\title{
Leishmaniasis canina: diagnóstico y manejo terapéutico
}

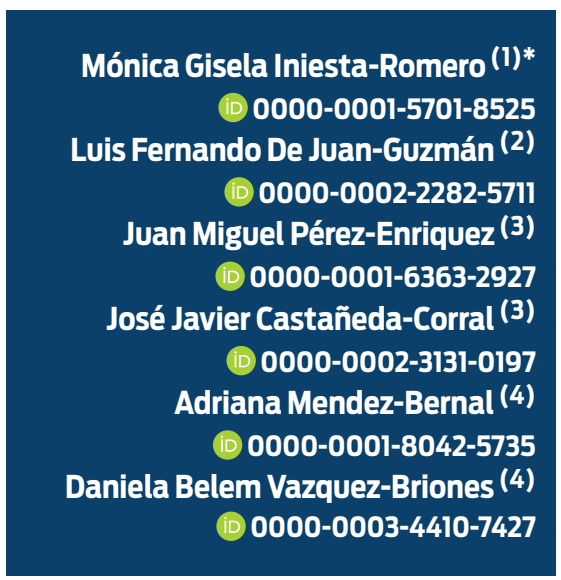

(1)Universidad Nacional Autónoma de México. Facultad de Medicina Veterinaria y Zootecnia. Hospital Veterinario de Especialidades.

(2) Universidad Nacional Autónoma de México. Facultad de Medicina Veterinaria y Zootecnia. Departamento de Medicina, Cirugía y Zootecnia para Pequeñas Especies.

(3) Universidad Nacional Autónoma de México. Facultad de Medicina Veterinaria y Zootecnia. Departamento de Patología, área Patología Clínica.

(4) Universidad Nacional Autónoma de México.

Facultad de Medicina Veterinaria y Zootecnia. Departamento de Patología, área de Anatomopatología.

* Autor para correspondencia: Correo electrónico: *mvz.iniesta89@outlook.com

Recibido: 2019-05-24 Aceptado: 2019-10-07 Publicado: 2019-12-11

Información y declaraciones adicionales en la página 9

() Derechos de autor: Mónica Gisela Iniesta-Romero et al. 2019

acceso abierto $\mathbf{O}$

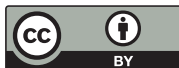

Distribuido bajo una Licencia Creative Commons Atribución 4.0 Internacional (CC-BY 4.0)

\section{Resumen}

Descripción del caso clínico: perro de 3.6 años de edad que acude a consulta con historia de secreción nasal, epistaxis y lesiones dermatológicas y diarrea con un mes de evolución.

Hallazgos clínicos: en el examen físico general se observó secreción nasal serosa en ambas narinas, nódulo en la región del maxilar del lado derecho y linfadenomegalia poplítea. En la bioquímica sérica, se reportó hiperglobulinemia de 104 g/L (23 - 39); en la electroforesis de proteínas: incremento de gammaglobulinas asociado a una gammapatía y se aspiró con aguja delgada (ACAD) de linfonodo poplíteo, y en su análisis se encontraron estructuras parasitarias compatibles con amastigotes de Leishmania spp.

Tratamiento y evolución: se instauró tratamiento médico con alopurinol, pentoxifilina y antimoniato de meglumina, éste último, únicamente por cuatro semanas debido a la falta de su distribución en México; con lo que se logra mantener a la paciente sin signos clínicos. La anemia se asocia a la inflamación crónica, por lo que se administró vitamina B12.

Pruebas complementarias de laboratorio: Bioquímica sérica en cada consulta para evaluar función renal, electroforesis de proteínas y aspirado con aguja delgada (ACAD) de linfonodo poplíteo

Relevancia clínica: la leishmaniasis canina es una enfermedad poco diagnosticada en México, los signos clínicos que se presentan son inespecíficos, por

Una forma de citar este artículo:

Iniesta-Romero MG, De Juan-Guzmán LF, Pérez-Enriquez JM, Castañeda-Corral JJ, Mendez-Bernal A, Vazquez-Briones DB. Leishmaniasis canina: diagnóstico y manejo terapéutico. Clínica veterinaria: abordaje diagnóstico y terapéutico. 2019;5:e45201959. DOI: 10.22201/fmvz.23958766e.201945. 
lo que no se da el tratamiento adecuado oportunamente y puede existir alta mortalidad de los pacientes con esta enfermedad. Es de importancia clínica saber que existen regiones en México en donde habitan especies de vectores que pueden transmitir la enfermedad.

Palabras clave: perro de agua español, zoonosis, flebótomo, enfermedad endémica, leishmaniasis en perros.

\section{Canine leishmaniasis: diagnosis and therapeutic management}

\section{Abstract}

Case report: a canine patient of 3.6 years of age attending consultation with a history of nasal discharge, epistaxis and dermatological lesions and diarrhea with a month of evolution.

Clinical findings: in general physical examination, serous nasal secretion was observed in both nostrils, nodule in the maxillary region of the right side and popliteal lymphadenomegaly. In serum biochemistry were reported $104 \mathrm{~g} / \mathrm{L}$ hyperglobulinemia (23 - 39); in protein electrophoresis, increased gammaglobulins associated with a gammapaty; the popliteal lymphonode was aspirated with fine needle and in its analysis were found parasitic structures compatible with amastigotes of Leishmania spp.

Treatment and evolution: medical treatment with allopurinol, pentoxifylline and meglumine antimoniate, the latter, was instituted for only four weeks due to the lack of its distribution in Mexico, thus maintaining the patient without clinical signs. Anemia is associated with the chronic inflammation process, so vitamin B12 was administered.

Laboratory tests: serum biochemistry in each consultation to evaluate renal function, protein electrophoresis and fine needle aspiration of popliteal lymphonode.

Clinical relevance: canine leishmaniasis is a poorly diagnosed disease in Mexico, the clinical signs that appear are nonspecific, therefore the adequate treatment is not given and there may be high mortality of patients suffering from the disease. It is of clinical importance to know that there are regions in Mexico where species of vectors that can transmit the disease live.

Keywords: Spanish Water Dog, nasal discharge, hypotrichosis, blepharitis, lymphadenomegaly, canine leishmaniasis 


\section{Descripción del caso}

Se trata a una perra de agua española, entera de 3.6 años. Fue adquirida en un criadero de Málaga, España, a los tres meses de edad y fue trasladada del criadero a la zona sur de la Ciudad de México, con calendario de vacunación y desparasitación vigente. Nunca había tenido ningún problema de salud, sin embargo, tres meses antes de acudir al hospital comenzó con secreción nasal, estornudos frecuentes, secreción ocular, otitis bilateral, lesiones costrosas en la punta de ambas orejas y epistaxis. Acudieron con un médico veterinario zootecnista (MVZ) quien comenzó tratamiento con amoxicilina y ácido clavulánico durante quince días, pero no mejoró. Al mes de evolución, sus propietarios notaron que los linfonodos submandibulares habían aumentado de tamaño, tenía fiebre y alopecia periocular. El mismo MVZ realizó citología de los linfonodos preescapulares y el poplíteo del lado izquierdo, donde se reportó hiperplasia linfoplasmocítica. No instauró tratamiento. Como la paciente no mejoró, buscaron una segunda opinión.

\section{Hallazgos clínicos e interpretación}

En el examen físico general, se encontró epistaxis de ambas narinas (Figura 1), linfadenomegalia generalizada (Figura 2), nódulo firme en el lado derecho del maxilar, de $1.4 \mathrm{~cm} \times 1.6 \mathrm{~cm}$, no desplazable, de bordes regulares, subcutáneo, no doloroso. Lesiones costrosas en la punta de las orejas, secreción ocular, otitis bilateral, alopecia periocular y en los corvejones. Se realizó ACAD de linfonodos poplíteos y del nódulo en el lado izquierdo del maxilar, y se tomaron muestras sanguíneas.

En el hemograma se encontró anemia ligera no regenerativa por inflamación crónica, sólidos totales $>120 \mathrm{~g} / \mathrm{L}$ (60-75 g/L), lo que concuerda con las proteínas totales de la bioquímica sérica de $120 \mathrm{~g} / \mathrm{L}(56-75 \mathrm{~g} / \mathrm{L})$, albúmina $16 \mathrm{~g} / \mathrm{L}$ (20$40 \mathrm{~g} / \mathrm{L})$ y globulinas de $104 \mathrm{~g} / \mathrm{L}(23-39 \mathrm{~g} / \mathrm{L})$, asociada a hiperproteinemia por hiperglobulinemia marcada e hipoalbuminemia por inflamación crónica, incremento de aspartato aminotransferasa (AST) $89 \mathrm{U} / \mathrm{L}(<55 \mathrm{U} / \mathrm{L})$ y CK $353 \mathrm{U} / \mathrm{L}(<213)$ por actividad o esfuerzo muscular sin descartar probable miositis por presencia de formas parasitarias y una hiperamilasemia e hipotrigliceridemia sin asociación clínica. El urianálisis arrojó hematuria marcada, que se asoció al método de obtención de la muestra (cistocentesis).

Por la hiperglobulinemia marcada 104 g/L (23-39 g/L) en la bioquímica sérica, se determinaron las fracciones proteicas mediante electroforesis, los resultados fueron albúmina $27.2 \%=44.9 \mathrm{~g} / \mathrm{L}(18.8 \%=22.6 \mathrm{~g} / \mathrm{L})$, alfaglobulinas $6.8 \%=8.2 \mathrm{~g} / \mathrm{L}(5.75 \%-18.67 \mathrm{~g} / \mathrm{L})$, betaglobulinas $14.3 \%=17.2 \mathrm{~g} / \mathrm{L}(5.07 \%$ $-15.91 \mathrm{~g} / \mathrm{L})$, y gammaglobulinas $60.1 \%=72 \mathrm{~g} / \mathrm{L}(2.26 \%-10.7 \mathrm{~g} / \mathrm{L})$.

El marcado incremento de las gammaglobulinas confirmó una gammapatía, lo que corresponde a mayor concentración de inmunoglobulinas de una sola región, donde se registra un pico alto de base delgada similar a la albúmina en el trazado del densitómetro, y se interpreta como una gammapatía monoclonal. ${ }^{1}$

La proliferación clonal de células neoplásicas de la serie linfoide B produce la mayoría de las gammapatías monoclonales, sin embargo, también se ha observado en pacientes con incremento plasmático no neoplásico, como en la ehrlichiosis canina, gastroenterocolitis plasmocítica y leishmaniasis. Hasta este momento, los diagnósticos diferenciales eran linfoma multicéntrico, mieloma múltiple, infección 


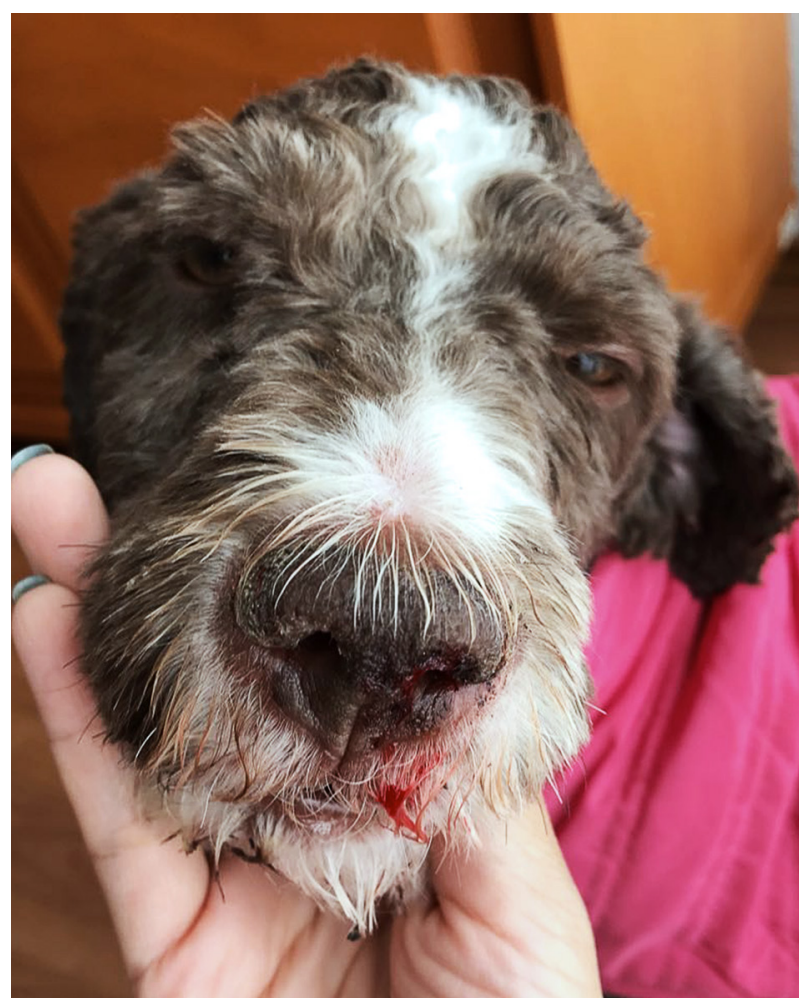

Figura 1. Epistaxis bilateral.

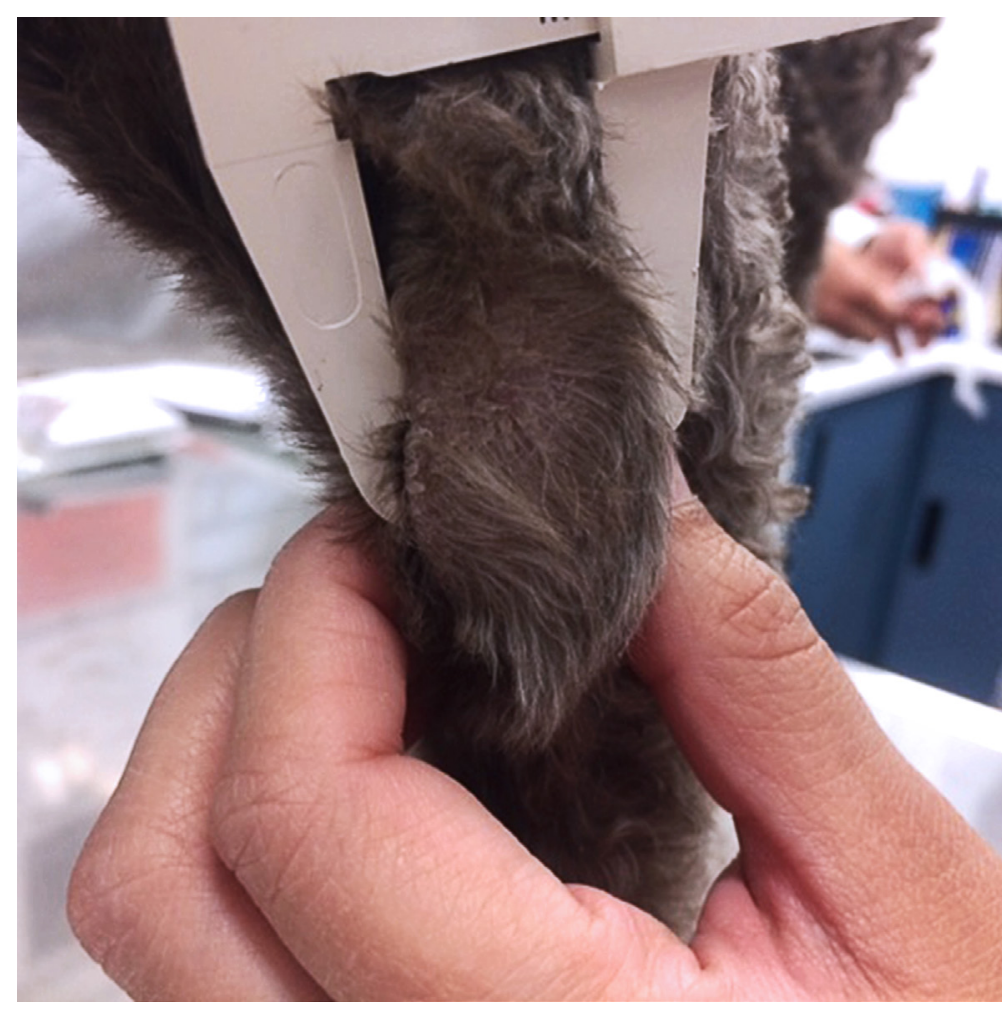

Figura 2. Linfadenomegalia generalizada. 
por Ehrlichia spp. Se puncionaron con ACAD los linfonodos poplíteo (Figura 3) y preescapular izquierdo, así como un nódulo cutáneo de $1.2 \mathrm{~cm}$ de eje mayor situado en el belfo derecho.

En el examen citológico, sobre un fondo de eritrocitos se observaron abundantes linfocitos en diversos estadios de maduración, así como moderados histiocitos, células plasmáticas, y escasos neutrófilos. En el citoplasma de los histiocitos, se apreciaron numerosas estructuras parasitarias compatibles con amastigotes de Leishmania spp. (Figura 4), las cuales también se vieron extracelularmente. El diagnostico citológico fue "linfadenitis histiocítica con amastigotes intraistiocíticos de Leishmania spp."

En el hemograma de la paciente, encontramos al indicio clínico más frecuente, la anemia. También hallamos a la característica hiperproteinemia marcada, misma que reduce los diagnósticos diferenciales. Este hallazgo fue confirmado en la bioquímica sérica y mediante electroforesis, se determinó una gammapatía monoclonal.

Se debe hacer una buena investigación en la historia clínica y reseña de los pacientes que provienen de zonas endémicas de este tipo de enfermedades, ya que a veces los signos clínicos pueden ser inespecíficos, lo cual demora el diagnótico y se instauran tratamientos inapropiados.

Los hallazgos citológicos muestran una respuesta inflamatoria compuesta principalmente por histiocitos y células plasmáticas, esto indica que hay antígeno. Como parte de la respuesta humoral, se expresan linfocitos B circundantes, por lo que se puede inferir que la paciente mantiene una apropiada respuesta inmune ante el microorganismo infectante. El diagnóstico citológico es la prueba diagnóstica rápida y contundente en casos con cuadros clínicos de leishmaniosis.

\section{Tratamiento y evolución}

Debido a los hallazgos compatibles con leishmaniasis canina, se propone a los propietarios comenzar tratamiento con antimoniato de meglumina, porque es el tratamiento de elección en España; sin embargo, no se vende en México, por lo que se mandó a casa medicada con allopurinol a $10 \mathrm{mg} / \mathrm{kg}$ PO BID. Por la epistaxis asociada a síndrome de hiperviscosidad sanguínea, se agregó pentoxifilina a $10 \mathrm{mg} / \mathrm{kg}$ PO TID. Mes y medio después se administró el antimoniato de meglumina.

Se realizó ultrasonido abdominal para descartar enfermedad visceral alguna, no se encontró ninguna alteración. Actualmente la paciente se encuentra estable, las alteraciones dermatológicas han disminuido considerablemente, pero se continuará monitoreando con estudios de laboratorio.

Respecto a las alteraciones asociadas al proceso de inflamación crónica, incluyendo la hipoalbuminemia y la anemia moderada, se suplementó a la paciente con albúmina oral en polvo y vitamina B12.

\section{Pruebas de laboratorio}

Se realizó hemograma, bioquímica sérica y urianálisis en cada consulta, y una sola determinación de fracciones proteicas, las alteraciones y los resultados son los interpretados en la sección de hallazgos clínicos e interpretación. 


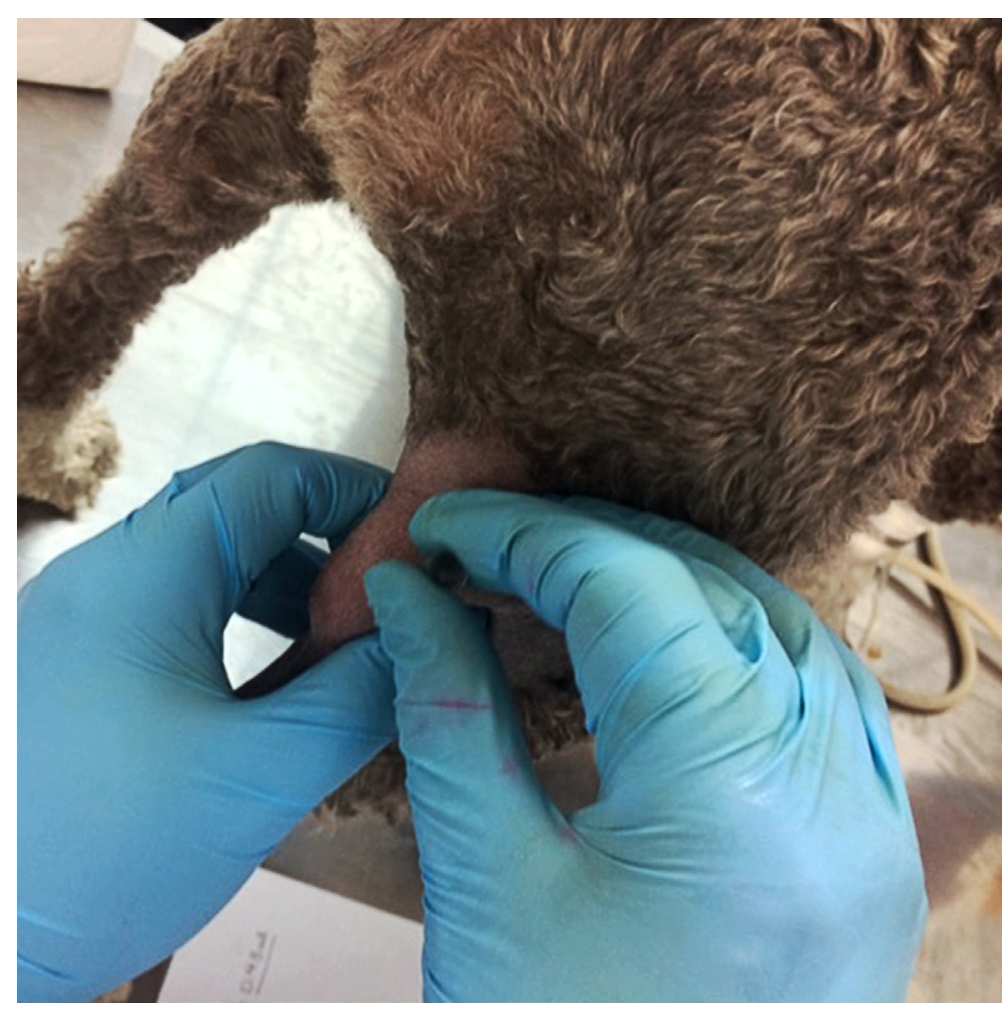

Figura 3. ACAD (Aspirado con aguja delgada) linfonodos poplíteos.

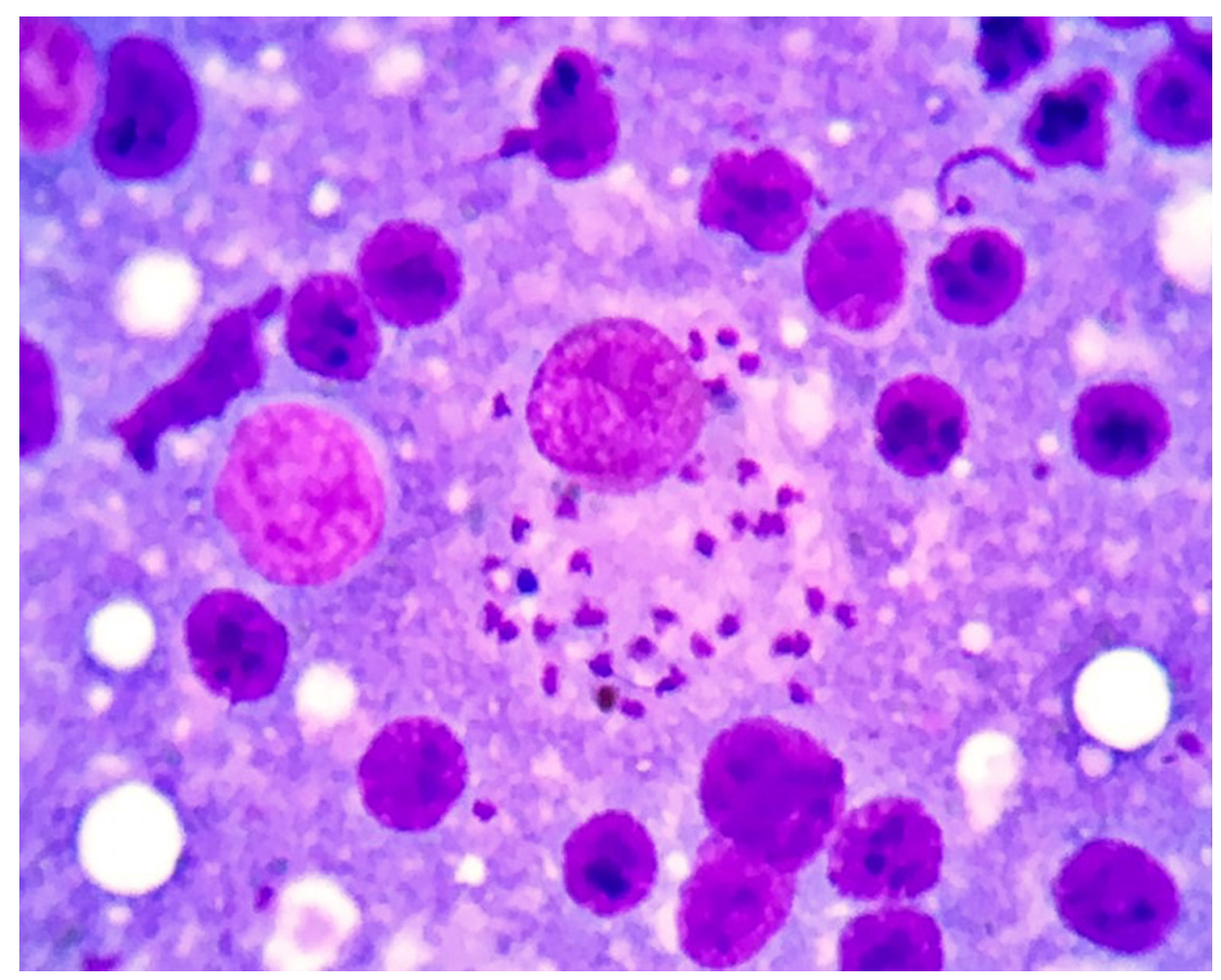

Figura 4. Amastigotes de Leishmania spp. 


\section{Discusión y relevancia clínica}

La leishmaniasis pertenece a un grupo de enfermedades causadas por especies del protozoo Leishmania, de la familia Tripanosomatidae. Se considera un parásito intracelular obligado de los mamíferos, entre ellos el humano. La infección es endémica en grandes áreas tropicales, subtropicales y en la cuenca del Mediterráneo. ${ }^{2}$

Es una enfermedad prevalente en más de 98 países. En México se han reportado casos aislados, generalmente en zonas agrícolas, en donde hay siembra o cosecha de cacao o café. Algunos de estos lugares corresponden a tres regiones, la del Golfo: Veracruz, Tabasco, Campeche, Quintana Roo y Yucatán; la del Pacífico: Chiapas, Guerrero, Jalisco, Nayarit, Oaxaca y Sinaloa; y la del Centro: Morelos y Puebla.

El género Leishmania contempla más de veinte especies que se dividen en tres subgéneros: Leishmania, Viannia, Sauroleishmania. La clasificación se realiza de acuerdo con su biología (desarrollo en el flebótomo, crecimiento en medios de cultivo, desarrollo en los hospederos vertebrados); bioquímica (patrones isoenzimáticos); inmunología (análisis parasitario con anticuerpos monoclonales) y filogenia molecular (secuenciación de múltiples loci). ${ }^{2}$

La leishmaniasis es sensible a las condiciones climáticas, que afectan en varios aspectos su epidemiología. Se ha visto que los cambios de temperatura, precipitaciones y humedad tienen efectos importantes en los vectores y reservorios animales. Las pequeñas fluctuaciones en la temperatura afectan el ciclo de desarrollo de promastigotes de leishmania y permiten que el parásito se transmita en zonas donde la enfermedad no era endémica. ${ }^{2}$

La enfermedad se manifiesta principalmente de tres formas: leishmaniasis visceral, en más del $25 \%$ de los casos es mortal si no se trata; leishmaniasis cutánea, se produce en zonas expuestas principalmente a uniones mucocutáneas, y genera lesiones ulcerativas; y leishmaniasis mucocutánea, que conduce a la destrucción parcial o completa de las membranas mucosas de la nariz, boca y garganta.

Se han descrito diferentes signos, dentro de estas alteraciones generalmente se reportan lesiones cutáneas como dermatitis exfoliativa, focal o generalizada con alopecia que evoluciona a dermatitis ulcerativa. El microorganismo inoculado por el insecto hematófago es fagocitado por los histiocitos, donde prolifera activamente causando destrucción de más células infectadas; los protozoarios liberados son nuevamente fagocitados por células mononucleares fagocíticas y el proceso se repite.

Cuando la inflamación se extiende a los epitelios, se ulceran especialmente las prominencias óseas y las uniones mucocutáneas. ${ }^{3}$ Es frecuente la linfadenomegalia asociada a una respuesta inmune contra el parásito. El periodo de patencia y prepatencia de la enfermedad varía de tres a dieciocho meses. Se ha documentado que el periodo de latencia incluso permanece años. ${ }^{4}$

No todos los perros infectados por Leishmania desarrollan la enfermedad debido a las respuestas inmunes montadas, las cuales determinarán si progresará a un estado subclínico o a una enfermedad clínica. Es importante realizar pruebas de PCR para determinar la especie de leishmania; sin embargo, esto no presume el tipo de expresión clínica que el paciente desarrollaría; aunque el tratamiento no se modifique, es posible que el paciente únicamente esté infectado por una especie de Leishmania. 
El grado de hiperproteinemia por hiperglobulinemia es muy importante, pues son pocos los diagnósticos diferenciales para este tipo de hallazgos de laboratorio. Entre los principales diferenciales hay otras causas linfoproliferativas, la más frecuentemente es el sarcoma de plasmocitos (mieloma múltiple), donde las principales proteínas implicadas son las inmunoglobulinas IgM e IgA, y la macroglobulinemia de Waldenstrom, cuya paraproteinemia se relaciona con una crioglobulina. Consideramos otros diagnósticos como la leucemia linfocítica crónica, el plasmocitoma cutáneo y el linfosarcoma. De origen infeccioso, los principales diagnósticos diferenciales son la ehrlichiosis y, por supuesto, la leishmaniasis, estas últimas, con más frecuencia presentan una gammapatía policlonal, aunque se han descrito casos de gammapatías monoclonales. 5,6

Para el diagnóstico citológico nos basamos en la identificación del microorganismo en preparaciones citológicas o histológicas, por aspirados de linfonodos, piel, médula ósea, bazo e hígado. Los hallazgos citológicos más característicos en un aspirado de linfonodo es una hiperplasia linfoide o una linfadenitis histiocítica, donde se aprecian los protozoarios dentro del citoplasma de los histiocitos. Una característica típica que se observa en el examen citológico son los cuerpos de DonovanLeishman, que corresponden a los kinetoplastos (DNA mitocondrial). Es importante realizar pruebas de PCR para determinar la especie de leishmania, sin embargo esto no establece el tipo de presentación clínica que el paciente desarrollaría.

En este caso, la primera vez que la paciente acudió con un MVZ fue porque presentaba lesiones nasales en las uniones mucocutáneas, atribuibles a lo antes mencionado. Pero, los signos clínicos más evidentes en esta paciente fueron debilidad y descarga ocular, reportados en el tipo visceral, resultado de una pobre respuesta inmunitaria, ya que si no se comienza tratamiento, los macrófagos infectados se diseminan en todo el organismo, principalmente en bazo, linfonodos, hígado y riñones.

En esta paciente se realizó ultrasonido abdominal, en él no se encontraron alteraciones, solo sugirió que desencadenaría la versión cutánea de leishmaniasis. ${ }^{3,7}$ De acuerdo con Huebner et al. (2008), en Madrid, España, se han encontrado infecciones por L. infantum en $3.7 \%$ de gatos $y, 3.2 \%$ de perros. Mientras, al sur de España, de donde proviene la paciente, se reportan $28.3 \%$ de casos. $^{8}$

Es importante el reporte de estos casos, ya que no existe suficiente investigación acerca de la prevalencia de Leishmania spp. en la población canina del territorio nacional. De manera sustancial, es obligación de los MVZ ampliar los diagnósticos diferenciales así como los estudios diagnósticos para brindar mayores oportunidades de recibir tratamiento adecuado a los infectados. 


\section{Financiamiento}

Pruebas de imagen, histopatología y patología clínica financiadas por el propietario.

\section{Conflictos de interés}

Los autores de este trabajo declaran no tener conflicto de interés.

\section{Contribución de los autores}

MGIR. Diagnóstico clínico.

LFJG, JCC, MGIR. Análisis, integración y redacción.

MGIR. Diagnóstico clínico.

JMPE, JCC. Diagnóstico patológico.

DBVB, AM. Diagnóstico anatomopatológico.

\section{Referencias}

1. Núñez Ochoa L, Bouda J. Patología clínica veterinaria. México, DF: UNAM; 2007. $348 \mathrm{p}$.

2. Akhoundi M, Downing T, Votýpka J, Kuhls K, Lukeš J, Cannet A, et al. Leishmania infections: molecular targets and diagnosis. Mol Aspects Med. octubre de 2017;57:1-29.

3. Pace D. Leishmaniasis. J Infect. noviembre de 2014;69 Suppl 1:S10-18.

4. Font A, Closa JM, Mascort J. Monoclonal gammopathy in a dog with visceral leishmaniasis. J Vet Intern Med. junio de 1994;8(3):233-5.

5. Kiral FK, Seyrek K, Pasa S, Ertabaklar H, Ünsal C. Some haematological, biochemical and electrophoretic findings in dogs with visceral leishmaniasis. Revue Méd Vét. 2004;4:226-9.

6. Antognoni MT, Birettoni F, Miglio A, Lalli P, Porciello F, Pecci VM. Monoclonal gammopathy associated with multiple myeloma and visceral leishmaniasis in the dog: a comparison of two cases. Vet Res Commun. 2010;34(S1):97-101. doi: 10.1007/s11259-010-9365.

7. Kima PE. Leishmania molecules that mediate intracellular pathogenesis. Microbes Infect. septiembre de 2014;16(9):721-6.

8. Mylonakis ME, Papaioannou N, Saridomichelakis MN, Koutinas AF, Billinis C, Kontos VI. Cytologic patterns of lymphadenopathy in dogs infected with Leishmania infantum. Vet Clin Pathol. septiembre de 2005;34(3):243-7. 